\title{
Finite-Difference and Pseudospectral Time-Domain Methods Applied to Backward-Wave Metamaterials
}

\author{
Michael W. Feise, John B. Schneider, Member, IEEE, and Peter J. Bevelacqua
}

\begin{abstract}
Backward-wave (BW) materials that have simultaneously negative real parts of their electric permittivity and magnetic permeability can support waves where phase and power propagation occur in opposite directions. These materials were predicted to have many unusual electromagnetic properties, among them amplification of the near-field of a point source, which could lead to the perfect reconstruction of the source field in an image [J. Pendry, Phys. Rev. Lett. vol. 85, pg. 3966, 2000]. Often systems containing BW materials are simulated using the finite-difference time-domain technique. We show that this technique suffers from a numerical artifact due to its staggered grid that makes its use in simulations involving BW materials problematic. The pseudospectral time-domain technique, on the other hand, uses a collocated grid and is free of this artifact. It is also shown that when modeling the dispersive $B W$ material, the linear frequency approximation method introduces error that affects the frequency of vanishing reflection, while the auxiliary differential equation, the $Z$-transform, and the bilinear frequency approximation method produce vanishing reflection at the correct frequency. The case of vanishing reflection is of particular interest for field reconstruction in imaging applications.
\end{abstract}

Index Terms-Backward-wave (BW) material, double-negative material, finite-difference time-domain (FDTD) methods, left-handed material, metamaterial, pseudospectral time-domain (PSTD) method.

\section{INTRODUCTION}

$\mathbf{E}$ LECTROMAGNETIC metamaterials with simultaneously negative electric permittivity and magnetic permeability have recently received much attention. These materials can support a backward-traveling wave where the phase propagation is antiparallel to the direction of energy flow. These materials have been identified by several names including left-handed or double-negative material but we shall refer to them here as backward-wave (BW) materials. Their properties were first considered theoretically by Veselago [1] during the 1960s but they have only been fabricated recently [2], [3]. They are predicted to exhibit many unusual properties such as refraction at a negative angle, an inverse Doppler shift,

Manuscript received April 17, 2003; revised January 29, 2004. This work was supported by the Office of Naval Research code 321OA. This work performed at the School of Electrical Engineering and Computer Science, Washington State University, Pullman, WA.

M. W. Feise is with the Nonlinear Physics Centre, Research School of Physical Sciences and Engineering, Australian National University, Canberra, ACT 0200, Australia.

J. B. Schneider is with the School of Electrical Engineering and Computer Science, Washington State University, Pullman, WA 99164 USA (e-mail: schneidj@eecs.wsu.edu)

P. J. Bevelacqua is with Stanford University, Stanford, CA 94305 USA.

Digital Object Identifier 10.1109/TAP.2004.835274 and a backward oriented Čerenkov radiation cone [1]. Veselago [1] also predicted that, under the conditions

$$
\varepsilon_{r}=\mu_{r}=-1
$$

a parallel slab of this material could focus waves emitted from a point source and essentially act as a lens, albeit without magnification. These conditions are considered "ideal" because they lead to the same impedance and speed of light as free space.

Pendry [4] pointed out that under these conditions incident decaying evanescent waves become growing inside the slab. The recovery of the evanescent waves allows the image resolution to be better than the diffraction limit and this material could theoretically produce a perfect image of the source, which lead Pendry to call the system a "perfect lens." A BW material must necessarily be dispersive [1] and therefore all this would only work properly at certain frequencies, where the conditions of (1) are fulfilled. At these frequencies the metamaterial slab not only compensates for the phase propagation of the waves, as a conventional lens does, but also compensates for the amplitude decay experienced by evanescent waves.

This claim of growing evanescent waves and the possibility of a perfect lens has aroused much interest and given rise to much discussion of its correctness and feasibility, e.g., [5]-[12]. Besides that, there is great interest and potential for applications in these materials beyond the issue of a perfect lens.

In this paper we study the behavior of an evanescent wave interacting with a slab of BW material via simulations. We consider the performance and applicability of two numerical techniques being used to simulate this interaction. In Section II we introduce the model system and in Section III the numerical schemes. Section IV discusses results and Section V presents conclusions.

\section{MODEL SySTEM}

To be able to study the properties of evanescent waves interacting with a BW slab, we isolate a single evanescent wave by inserting the slab into a parallel plate waveguide and use excitation below the cutoff frequency of the waveguide. This way the wave in the waveguide is evanescent and its wave vector can be chosen through the width of the waveguide and the spatial profile of the source. Fig. 1 shows a schematic of the system. The top and bottom of the waveguide are perfectly electrically conducting (PEC) plates. PEC walls also exist at the sides of the computational domain but these are behind perfectly-matched layers (PML) which essentially allow the structure to mimic an open waveguide (but the evanescent fields considered here 


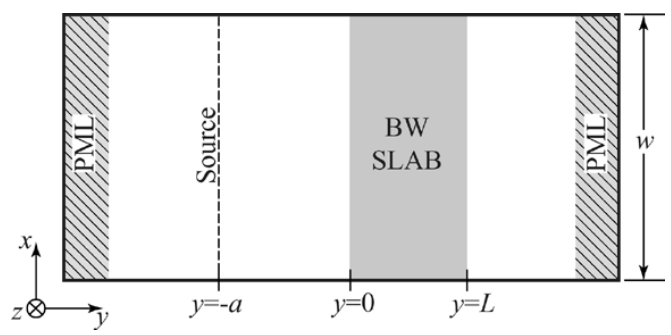

Fig. 1. Schematic view of the model system. The parallel plate waveguide with width $w$ is translationally invariant in the $z$ direction. A sheet current source at $y=-a$ and a slab of BW material are located inside the waveguide.

decay rapidly enough that the performance of the PML is not a critical component in the simulation). The system is translationally invariant in the $z$ direction and can be treated as two-dimensional (2-D). A sheet current density $\mathbf{j}$ at $y=-a$ parallel to the $x-z$ plane acts as the source for the fields. As our interest is in the behavior of evanescent fields, a single evanescent mode is excited using the spatial profile of a sheet current matched to that of the lowest order evanescent mode

$$
\mathbf{j}(x, y, t)=\hat{z} j(t) \delta(y+a) \sin \left(\pi \frac{x}{w}\right)
$$

where $\delta$ is the Dirac delta function and $w$ is the width of the waveguide. The source location and the waveguide width are shown in Fig. 1, and $j(t)$ is the time dependence of the current source. (In the steady-state situation we deal with a single or few wave vectors. For this reason the concern of [7] does not apply here.)

Composite materials in which the effective electric permittivity and the effective magnetic permeability are both negative in some frequency interval have experimentally been shown to exist [2], [3]. Based on this we assume that both the electric permittivity and the magnetic permeability are described by Lorentz-type frequency dependences as

$$
\begin{aligned}
& \varepsilon_{r}(\omega)=1+\frac{\omega_{e p}^{2}}{\omega_{e 0}^{2}+i \gamma_{e} \omega-\omega^{2}} \\
& \mu_{r}(\omega)=1+\frac{\omega_{m p}^{2}}{\omega_{m 0}^{2}+i \gamma_{m} \omega-\omega^{2}} .
\end{aligned}
$$

Here, $\omega_{e p}$ and $\omega_{m p}$ are the plasma frequency, $\omega_{e 0}$ and $\omega_{m 0}$ are the resonance frequency, and $\gamma_{e}$ and $\gamma_{m}$ are the absorption parameter of the permittivity and permeability, respectively. To simplify the model we choose $\varepsilon_{r}$ and $\mu_{r}$ to coincide, i.e., $\omega_{e p}=\omega_{m p}=\omega_{p}, \omega_{e 0}=\omega_{m 0}=\omega_{0}$, and $\gamma_{e}=\gamma_{m}=\gamma$. In the special case of $\omega_{0}=\gamma=0$, the conditions (1) are fulfilled at the design frequency

$$
f_{d}=\frac{\omega_{p}}{2 \pi \sqrt{2}}
$$

We take (5) to define the relationship between the design frequency and the plasma frequency even when $\omega_{0}$ and $\gamma$ are nonzero but small.

Our attention is primarily concerned with the design frequency since this is the only frequency at which a perfect focus could possibly be achieved. At a frequency other than the design frequency the rate at which evanescent fields grow in the slab is not equal to the rate they decay in free space. Thus for a source emitting multiple evanescent fields in front of the slab, there is no unique point on the other side of the slab at which the fields have obtained the same level as at the source (i.e., there is no unique image point).

We assume that the material has abrupt edges and the change in constitutive parameters is instantaneous and collocated. The case of the change in the constitutive parameters of a BW slab not coinciding leads to a shift in the frequency of the nonreflecting wave and the bound modes of the slab [13]. The fields of the bound modes decay exponentially away from the slab. The two bound modes with spatially decaying fields inside and outside the slab delimit a frequency interval that also contains a nonreflecting wave with exponential spatial dependence.

For the material reaction to the field to be causal, the Kramers-Kronig relations [14], [15] show that for any deviation from free-space behavior the imaginary part of the permittivity or permeability cannot vanish for all frequencies. On the other hand, as far as causality is concerned, it can be arbitrarily small.

\section{NUMERICAL SCHEMES}

The numerical schemes used here are applied directly to Maxwell's equations rather than to a wave equation. The first-order partial differential equations are discretized in time and space and an update algorithm is developed. Specifically the behavior of the traditional Yee finite-difference time-domain (FDTD) algorithm [16], [17] and the pseudospectral time-domain (PSTD) method [18] are studied. In both schemes the system is modeled as 2-D and the open sides of the waveguide are modeled by using a causal uniaxial perfectly matched layer (PML) absorbing boundary [19]-[21]. One significant difference between the two schemes lies in the grid location where the fields are sampled. In the PSTD scheme the sample points of the fields are collocated while in the FDTD scheme they are staggered.

The plates of the parallel plate waveguide are modeled as perfect electric conductors. On the PEC boundary, the tangential components of the electric field and the normal component of the magnetic field must vanish. The boundary conditions of the other components are given by surface charge density $\rho_{s}$ and surface current density $\mathbf{J}_{S}$,

$$
\begin{aligned}
\hat{\mathbf{n}} \times \mathbf{E} & =\mathbf{0} \\
\hat{\mathbf{n}} \cdot \mathbf{E} & =\frac{\rho_{s}}{\varepsilon} \\
\hat{\mathbf{n}} \times \mathbf{H} & =\mathbf{J}_{S} \\
\hat{\mathbf{n}} \cdot \mathbf{H} & =0 .
\end{aligned}
$$

Here, $\hat{\mathbf{n}}$ is the surface normal unit vector.

\section{A. Yee Scheme}

In 1966, Yee [16] proposed a discretization scheme for Maxwell's equations based on a fully staggered grid, where the electric field components are each sampled on one of the edges of a cubical unit cell and the magnetic components each on one of the face centers. In this manner the spatial derivatives can be approximated as centered differences and one achieves second-order accuracy in the spatial step size [17]. 
The PEC boundary conditions are implemented by placing the boundary in a plane that contains the tangential electric field sampling points and then setting these components to zero. In this case, the tangential magnetic field components and the normal electric field component are not sampled on the boundary and one does not need to enforce any boundary condition on them. The normal component of the magnetic field is sampled on the boundary and is updated in the usual fashion. Its boundary condition is automatically fulfilled through its dependence on the tangential electric field.

Owing to the staggering of the field components, the precise location of an interface where both the permittivity and permeability change is ambiguous. The case where a single material parameter, such as the permittivity, changes has been well-studied in the literature, e.g., [22], [23]. It is well established that for a tangential field component collocated with the interface, the best approach is to use the arithmetic average of the material properties to either side. (It can also be shown, at least for the case of normal incidence, that if one is free to assign the material interface to fall halfway between tangential nodes, an abrupt change in the material parameters is optimum and, in fact, superior to averaging [24].) For the study here, the precise location of the interface is not a primary concern. Instead, the important issue is whether the evanescent fields exhibit the proper growth within the BW slab (whatever its thickness or location). As reported in [25], averaging of material parameters has been used for modeling BW slabs with the Yee algorithm. (In that work surface waves are present and no subwavelength imaging is seen.)

Fig. 2 shows a portion of the computational grid as well as the material properties associated with each node-one must attribute a permeability to each magnetic field node and a permittivity to each electric field node. Fig. 2(a) shows the case of using abrupt discontinuities in the material parameters in the Yee grid while Fig. 2(b) shows the case where averaging is used for the tangential node at the interface. Fig. 2(c) shows the grid for the PSTD simulation which is described next.

\section{B. Pseudospectral Scheme}

In pseudospectral techniques a discrete Fourier transform is used to calculate the spatial derivatives in the discretized version of a partial differential equation. Time integration is achieved using the same second-order time-stepping approach as used in the Yee algorithm. Unlike the Yee algorithm where central-differences are used, in PSTD the spatial derivatives are calculated at the same location as the field. As shown in Fig. 2(c), for Maxwell's equations this means that the electric and the magnetic field components are all sampled at the same location, i.e., in the center of a cubic unit cell. An important problem with this approach is that the use of exponential Fourier transforms inherently introduces periodic boundary conditions that are often undesired. This problem can be avoided by covering the numerical domain boundaries with a PML layer such that the waves are absorbed in the PML before they can contaminate the simulation through the periodic boundaries [18]. This gives one a tool to simulate open domains. If the system contains PEC boundaries, the problem becomes much more difficult. Nonetheless,

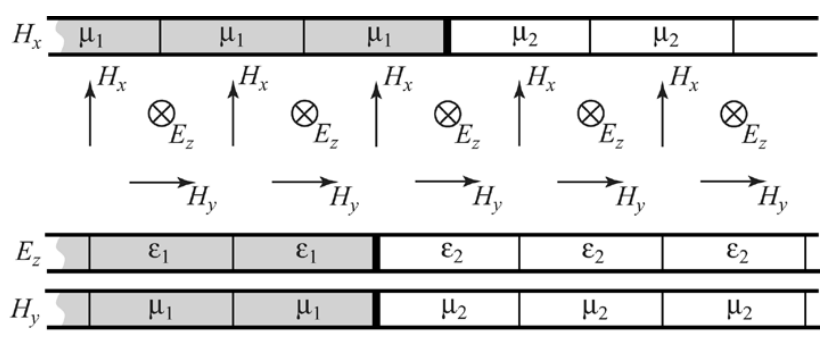

(a)

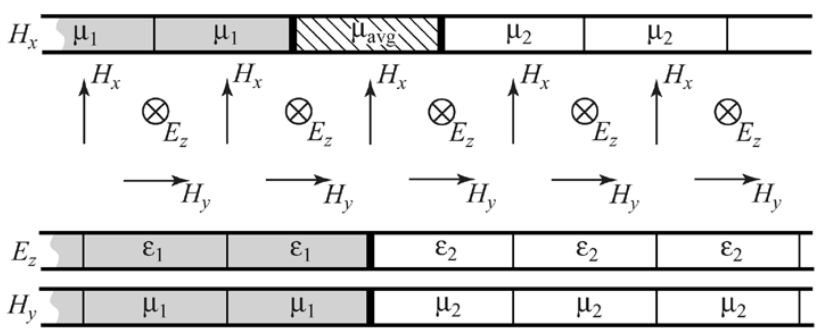

(b)

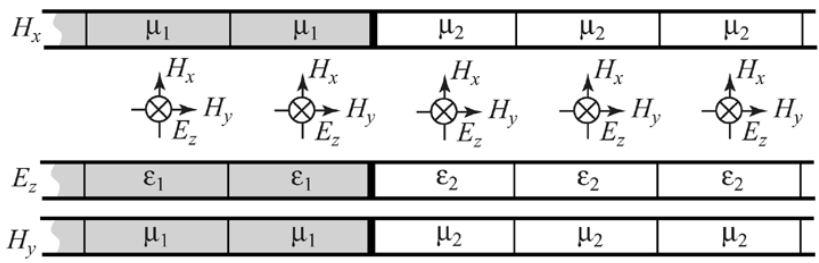

(c)

Fig. 2. Depiction of the way the permittivity and permeability change at an interface. The horizontal bars show the material parameters associated with a particular field component (the associated field is indicated by the label to the left of the bar). A slice of the grid indicating the node positions is given for each of the cases. (a) The Yee grid with "abrupt" boundaries. The interface is assumed to exist between the last $H_{x}$ which uses permeability $\mu_{1}$ and the first $E_{z}$ node which uses permittivity $\epsilon_{2}$. (b) The Yee grid using averaging. The interface is assumed to be collocated with the $H_{x}$ node which uses the average permittivity to either side even though the introduction of a new material $\left(\mu_{\text {avg }}\right)$ arguably creates an additional interface further to the left. (c) The PSTD grid. Because the grid is not staggered, the boundary between the two regions is unambiguously assumed to exist between the nodes which employ the different material parameters

in a 2-D system in transverse electric (TE) polarization, i.e., $\mathbf{E}$ perpendicular to the plane, which is source-free near the boundaries, one can show that the PEC boundary conditions [(6)-(9)] imply that

$$
(\hat{\mathbf{n}} \cdot \nabla)(\hat{\mathbf{n}} \times \mathbf{H})=\mathbf{0} .
$$

Here, $\hat{\mathbf{n}}$ is the surface normal unit vector and $\hat{\mathbf{n}} \cdot \nabla$ is the derivative in that direction. Conditions (6) and (10) can be easily enforced with Fourier sine and cosine transforms if one chooses Fourier sine transforms for the tangential electric field components and Fourier cosine transforms for the tangential magnetic field components. No spatial derivatives of normal components appear and no boundary condition needs to be enforced on them. Thus, in a 2-D system in TE polarization, the PEC boundary conditions are conveniently implemented through the choice of Fourier transform.

The discrete Fourier transform has problems representing a delta function, discretized as a Kronecker delta, correctly. For this reason it is advantageous to use a spatially smoothed rather than a highly localized source [18] to represent the source screen 
of (2). This problem also appears when a sudden change in material parameters leads to a sudden change in the fields. In the simulations one observes wiggles or ripples on the signal that can become significant if the change in field is strong. This artifact is the Gibbs phenomenon associated with the Fourier transforms.

\section{Dispersion Implementation}

To achieve negative $\varepsilon$ and negative $\mu$ in a material, this material must necessarily be dispersive [1]. In our model system we chose Lorentz dispersion characteristics as given in (3) and (4), [2], [4]. There are several commonly used methods to implement frequency dependence in a time-domain algorithm, e.g., the auxiliary differential equation (ADE) method [26], the recursive convolution method [27], [28], and the Z-transform method [29]-[31]. In this study we use the ADE method, the Z-transform method, and two frequency-approximation methods [32]. Here we present the update for the electric field; analogous equations hold for the magnetic field. In the following, $\Delta_{t}$ will denote the temporal step size of the discretization scheme.

1) ADE Method: One approach to implement a frequency dependent response in a discrete time-domain method is to transform the frequency-domain constitutive parameters into the time domain and then approximate the derivatives with differences based on Taylor-series expansions [26]. The frequency dispersion (3) at any given electric field node inside the material slab is implemented as

$$
\begin{aligned}
E^{n}= & \left\{\left(\omega_{0}^{2} \Delta_{t}^{2}+\gamma \Delta_{t}+2\right) \frac{D^{n}}{\varepsilon_{0}}-4 \frac{D^{n-1}}{\varepsilon_{0}}\right. \\
& +\left(\omega_{0}^{2} \Delta_{t}^{2}-\gamma \Delta_{t}+2\right) \frac{D^{n-2}}{\varepsilon_{0}} \\
& \left.+4 E^{n-1}-\left[\left(\omega_{p}^{2}+\omega_{0}^{2}\right) \Delta_{t}^{2}-\gamma \Delta_{t}+2\right] E^{n-2}\right\} \\
& \times\left[\left(\omega_{p}^{2}+\omega_{0}^{2}\right) \Delta_{t}^{2}+\gamma \Delta_{t}+2\right]^{-1}
\end{aligned}
$$

where $D$ is the electric flux density which must be stored as a separate quantity. The superscripts on the fields denote the time step at which the field values are taken.

2) Z Transform Method: The Z-transform is essentially a more general version of the discrete Fourier transform. It is strictly based on a sampled signal and provides an exact transformation between sample domain and $\mathrm{Z}$ domain. For frequency dependencies of the Debye, plasma, and Lorentz type and even some types of nonlinearities it allows one to derive exact update algorithms (i.e., exact in the sampled sense) for the sampled signal [29]-[32]. The frequency dispersion of (3) is implemented as

$$
\begin{aligned}
E^{n}= & \frac{D^{n}}{\varepsilon_{0}}-\frac{\omega_{p}^{2}}{\beta} \Delta_{t} I^{n-1} \\
I^{n}= & 2 e^{-\alpha \Delta_{t}} \cos \left(\beta \Delta_{t}\right) I^{n-1}-e^{-2 \alpha \Delta_{t}} I^{n-2} \\
& +e^{-\alpha \Delta_{t}} \sin \left(\beta \Delta_{t}\right) E^{n}
\end{aligned}
$$

where $\alpha=\gamma / 2, \beta=\sqrt{\omega_{0}^{2}-\gamma^{2} / 4}$, and $I$ is an auxiliary field. This implementation is applicable for real $\beta$, i.e., when the system response function is a damped sine wave. When $\beta$ is imaginary, the character of the response function changes and the Z-transform has to be implemented differently [31].
3) Frequency Approximation Method: Another common method to derive discrete time-domain update equations for the frequency dependence of a system is frequency approximation. Here one starts with the frequency domain expression for the system response and makes the transition to the discretized time domain by replacing each occurrence of $i \omega$ by $\left(1-z^{-1}\right) / \Delta_{t}$, i.e., a linear backward difference where $z^{-1}$ represents a shift back in time of one temporal step [31], [32]. When the frequency dependence becomes complicated this method can make the derivation of update equations much easier than the Z-transform method. On the other hand, in general, while it does not significantly change the computational burden, it introduces new error. A bilinear approximation, $i \omega \rightarrow\left(2 / \Delta_{t}\right)\left(\left(1-z^{-1}\right) /\left(1+z^{-1}\right)\right)$, gives better accuracy but increases the computational burden [31], [32]. The frequency dispersion (3) using the linear approximation is implemented as

$$
\begin{aligned}
E^{n}= & \left\{\left(\omega_{0}^{2} \Delta_{t}^{2}+\gamma \Delta_{t}+1\right) \frac{D^{n}}{\varepsilon_{0}}\right. \\
& \left.-\left(\gamma \Delta_{t}+2\right) I^{n-1}+I^{n-2}\right\} \\
& \times\left[\left(\omega_{p}^{2}+\omega_{0}^{2}\right) \Delta_{t}^{2}+\gamma \Delta_{t}+1\right]^{-1} \\
I^{n}= & \left\{\omega_{p}^{2} \Delta_{t}^{2} E^{n}+\left(\gamma \Delta_{t}+2\right) I^{n-1}-I^{n-2}\right\} \\
& \times\left[\omega_{0}^{2} \Delta_{t}^{2}+\gamma \Delta_{t}+1\right]^{-1} .
\end{aligned}
$$

In the bilinear approximation the electric field is updated using

$$
\begin{aligned}
E^{n}= & \left\{\left(\omega_{0}^{2} \Delta_{t}^{2}+2 \gamma \Delta_{t}+4\right) \frac{D^{n}}{\varepsilon_{0}}-2 \omega_{p}^{2} \Delta_{t}^{2} E^{n-1}\right. \\
& \left.+\left(2 \omega_{0}^{2} \Delta_{t}^{2}-8\right) I^{n-1}+\left(\omega_{0}^{2} \Delta_{t}^{2}-2 \gamma \Delta_{t}+4\right) I^{n-2}\right\} \\
& \times\left[\left(\omega_{p}^{2}+\omega_{0}^{2}\right) \Delta_{t}^{2}+2 \gamma \Delta_{t}+4\right]^{-1} \\
I^{n}= & \left\{\omega_{p}^{2} \Delta_{t}^{2} E^{n}+2 \omega_{p}^{2} \Delta_{t}^{2} E^{n-1}+\omega_{p}^{2} \Delta_{t}^{2} E^{n-2}\right. \\
& \left.-\left(2 \omega_{0}^{2} \Delta_{t}^{2}-8\right) I^{n-1}-\left(\omega_{0}^{2} \Delta_{t}^{2}-2 \gamma \Delta_{t}+4\right) I^{n-2}\right\} \\
& \times\left[\omega_{0}^{2} \Delta_{t}^{2}+2 \gamma \Delta_{t}+4\right]^{-1} .
\end{aligned}
$$

In (12)-(17), $I$ is used as an auxiliary field.

\section{RESUlTS}

We have performed model calculations for a slab of $\mathrm{BW}$ material inside a waveguide using the geometry and the material described in Section II. We compare the results for the FDTD and the PSTD schemes, using the ADE method for the dispersion implementation. We also show a comparison of all four dispersion implementations of Section III-C with the PSTD scheme.

For the FDTD method, one commonly uses the Yee scheme [16] to derive the update equations for the fields. Because this scheme is based on a staggered grid, where all field components are sampled at different locations in a unit cell, it has inherent difficulties representing a collocated change in material parameters. This problem becomes clearly evident when modeling an interface between free space and a BW material.

In the PSTD method all the field components are sampled at the same location and no transition layer exists. We find that, unless the dispersion implementation introduces phase error, the 
bound modes and the nonreflecting wave appear at frequencies very close to those found in the continuous world.

In the simulations the design frequency $f_{d}$ is $15 \mathrm{GHz}$ and the spatial discretization level is 100 points per propagating free-space wavelength at the design frequency $\lambda_{f}$, i.e., $\Delta_{x}=$ $\Delta_{y}=\lambda_{f} / 100=c /\left(100 f_{d}\right)=0.1999 \mathrm{~mm}$. Using such a fine discretization is typically unnecessary when simulating propagating waves, where it is more customary to use approximately 20 points per wavelength, but because evanescent waves have rapid amplitude decay and also phase variations (transverse to the amplitude decay) that have wavelengths smaller than the propagating free-space wavelength, it can be necessary to use a finer discretization to model these waves accurately.

The slab length $L$ is $33 \Delta_{y}$, and the source is located a distance $a=30 \Delta_{y}$ in front of the BW slab. The waveguide width $w$ which determines the degree of evanescence was chosen to be $32 \Delta_{x}$. (For $w>\lambda_{f} / 2=50 \Delta_{x}$ the waves in the waveguide would be propagating.) The material parameters were chosen as $\omega_{p} /(2 \pi)=\sqrt{2} f_{d}, \omega_{0} /(2 \pi)=5 \mathrm{MHz}$, and $\gamma /(2 \pi)=5 \mathrm{MHz}$. The values of $\omega_{0}$ and $\gamma$ are small compared to $\omega_{p}$ and their influence on the results is weak. Even though (1) can only be approximately fulfilled, due to the loss and the nonzero resonance frequency, we will assume that the design frequency (5) is unchanged. The results for the Lorentz material are similar to those obtained for an unmagnetized plasma, i.e., $\omega_{0}=0 \mathrm{~Hz}$. The time dependence of the source current is a sinusoid with an exponential ramp $j(t)=\left(1-e^{-t / \tau}\right) \sin \left(2 \pi f_{s} t\right)$, with carrier frequency $f_{s}$ and the ramping governed by $\tau$. In the simulations we used $\tau=5000 \Delta_{t}$.

The graphs presented in this section show the spatial dependence of the magnitude of a certain frequency component of the temporal Fourier transform of the fields in the $y$ direction. The temporal Fourier transform was recorded between 15000 time steps and when the simulation stopped at a total of 67000 time steps. The solid vertical lines indicate the extent of the BW slab, the dotted vertical line indicates the source location, and the arrows show an arbitrarily chosen object location and its corresponding image location. Ideally, at the design frequency the BW slab compensates exactly for the free-space propagation and decay of incident waves corresponding to the thickness of the slab. Thus, referring to the geometry of Fig. 1, an object located at $y=-b$ with $b \leq L$, is imaged at $y=2 L-b$.

\section{A. Nonreflecting Wave}

In the Yee discretization scheme, all field components are sampled at different locations in a unit cell. The permittivity and permeability are usually also sampled at these locations. A change in material parameters at the interface between two materials, therefore, is extended in space and the algorithm essentially sees a thin transition layer, where some parameters are those of one material and the others are those of the other material. As discussed in Section III-A, the interface may be approximated with or without an average of the material properties to either side. We identify the approach without averaging as the "abrupt" boundary.

For a BW slab, the effect of such a transition layer between the change in $\varepsilon$ and the change in $\mu$ has previously been studied using a continuous-space model [13]. Our Yee simulations employing abrupt boundaries have transition layers with the

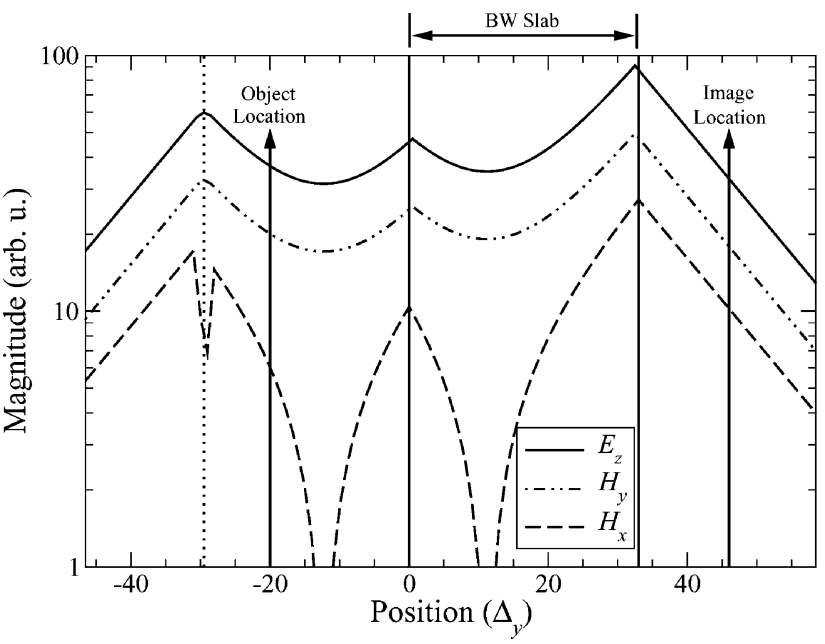

Fig. 3. Magnitude of the Fourier transform component at the design frequency using the abrupt-boundary Yee scheme with the ADE dispersion implementation. The lines for the different fields have been normalized and offset to allow qualitative comparison. The system parameters are given in the text. The solid vertical lines indicate the extent of the BW slab, the dotted vertical line indicates the source location and the arrows show an arbitrarily chosen object location and its corresponding image location.

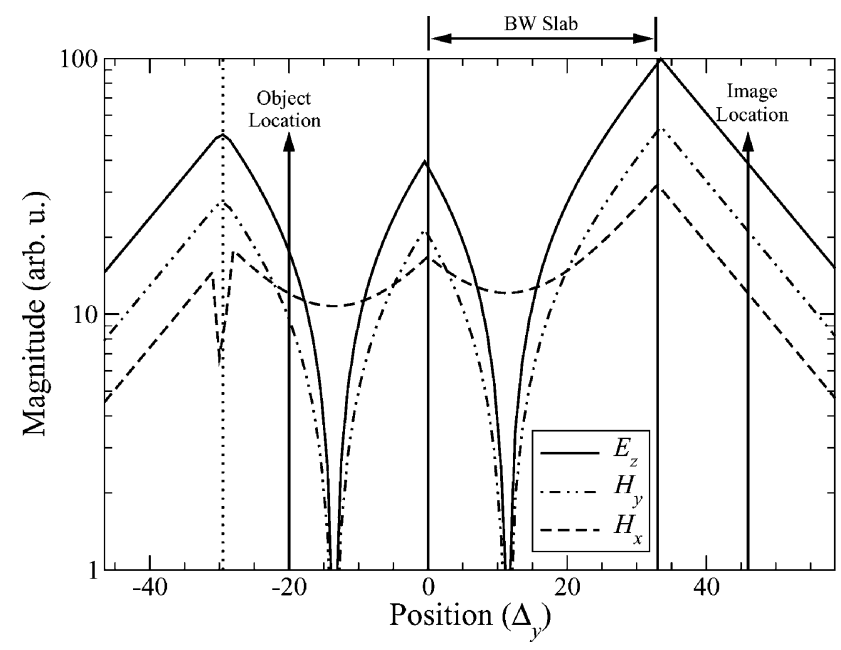

Fig. 4. Same as the previous figure except averaging is used for the permeability at the interface.

$\varepsilon$ of the BW slab and the $\mu$ of the surrounding free space, similar to the situation in [13]. For the front face of the slab, this corresponds to the situation shown in Fig. 2(a) if one associates the subscript 1 with free space and the subscript 2 with the BW material. When averaging is used, the average permeability is applied to the $H_{x}$ nodes assumed to coincide with the interfaces.

To illustrate the effect of the transition layer in the Yee scheme, we show in Fig. 3 the fields at the design frequency, where the source frequency $f_{s}$ equals $f_{d}$, using abrupt boundaries and the ADE dispersion implementation. The evanescent field grows inside the BW slab but a reflected field is clearly present, as can be seen from the change in slope of the fields between the source and the $y=0$ surface, as well as between the two slab surfaces. In Fig. 3 the field amplitudes at the image location are significantly different from those at the object location. Fig. 4 again shows the results at the design frequency 


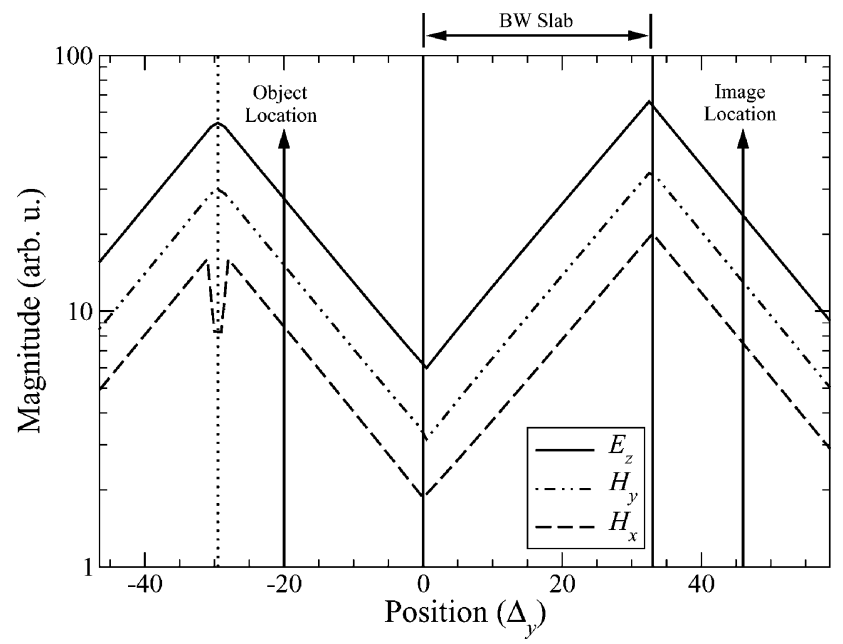

Fig. 5. Magnitude of the Fourier transform component at $f=14.83 \mathrm{GHz}$, away from the design frequency $f_{d}=15 \mathrm{GHz}$, using the abrupt-boundary Yee scheme with the ADE dispersion implementation. The lines for the different fields have been normalized and offset to allow qualitative comparison. The system parameters are given in the text.

except now averaging of the permeability is used at the interface. As before, the results do not exhibit the desired behavior. The deep nulls in both these figures occur when the total field changes sign because the reflected field is of equal magnitude as the field of the source at that point but has opposite sign.

Fig. 5 shows the nonreflecting wave in the Yee scheme with the abrupt boundary. It occurs at $14.83 \mathrm{GHz}$, i.e., below the design frequency $f_{d}$ of $15 \mathrm{GHz}$, and was found by scanning through the frequency range between the two bound mode frequencies. The field is clearly amplified exponentially inside the BW slab and decays exponentially outside of it. Upon close inspection one finds that the magnitude of the electric field at the object location is approximately 28 , while it is about 26 at the image location. This discrepancy is due to the slower speed of light in the BW material at this frequency. Thus, the exponential growth inside the slab is slower than the decay outside of it and, although the field is amplified in the slab, the amplification does not accurately compensate for the decay in free space. The magnitude of this discrepancy as well as the frequency at which the nonreflecting wave occurs is dependent on the wavevector of the field [13]. Thus, it is not possible to define a single corrected image location that holds for all wavevectors (this is true of both the propagating and evanescent components).

Fig. 6 shows the nonreflecting wave when averaging is used for the boundary. Here the nonreflecting wave was found to occur at $15.17 \mathrm{GHz}$, i.e., above the design frequency. An object of field strength 28 was found to have a corresponding field strength at the image point of 33 (i.e., the field was overcompensated).

In [13] it was found that transition layers shift the frequency of vanishing reflection coefficient upward from the design frequency. In the FDTD simulations we find that for the abrupt-boundary Yee scheme the nonreflecting frequency is shifted downward from the design frequency. We attribute this difference to the effect the discretization has on the transmission and reflection coefficient of each interface. (This is in addition to introducing transition layers into the system.) When averaging is used, the nonreflecting frequency is above the

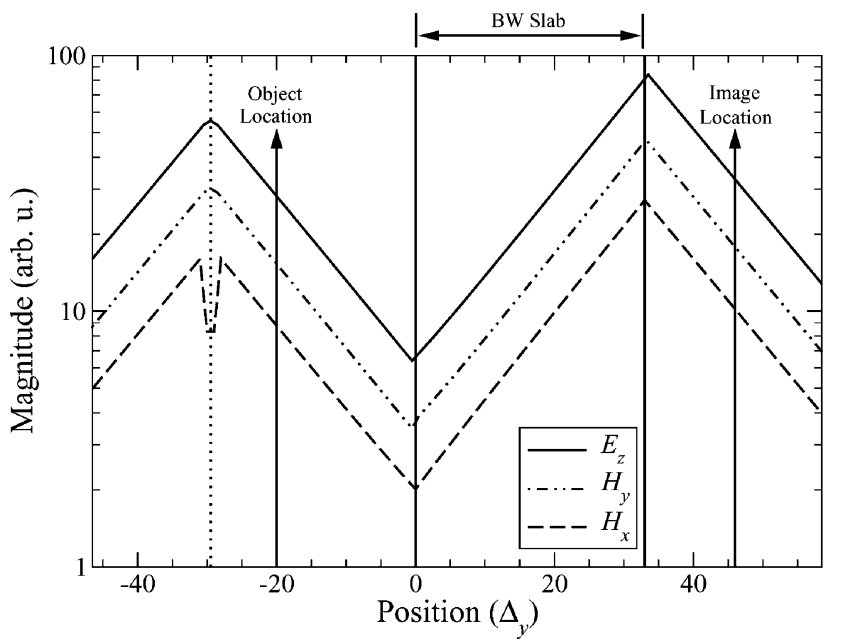

Fig. 6. Magnitude of the Fourier transform component at $f=15.17 \mathrm{GHz}$ using the Yee scheme with material averaging and the ADE dispersion implementation. The lines for the different fields have been normalized and offset to allow qualitative comparison. The system parameters are given in the text.

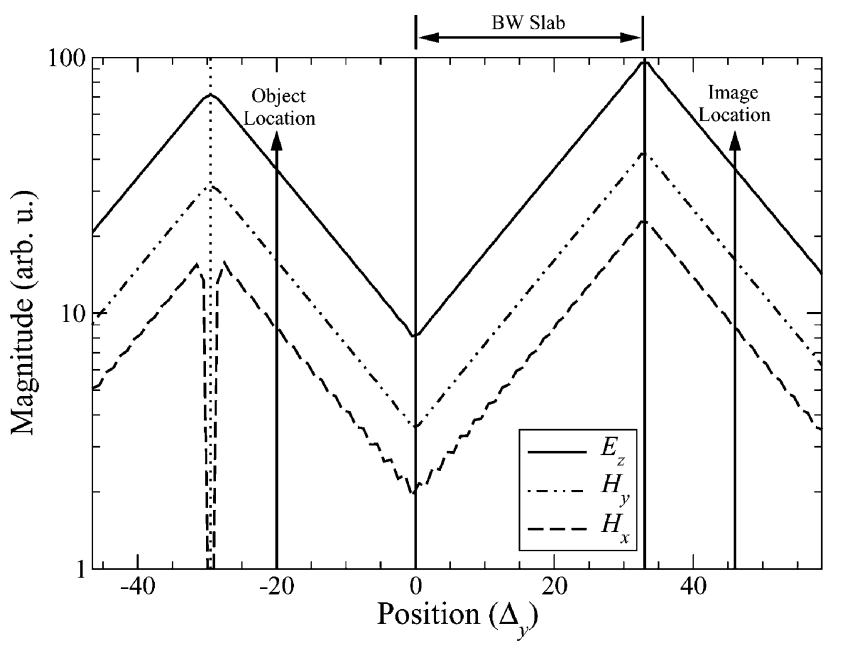

Fig. 7. Magnitude of the Fourier transform component at the design frequency using the PSTD scheme with the ADE dispersion implementation. The lines for the different fields have been normalized and offset to allow qualitative comparison. The system parameters are given in the text. The ripple on $H_{x}$ is a numerical artifact of the Fourier transform due to finite spatial resolution.

design frequency, but the offset between the two frequencies is approximately the same as when the abrupt boundary is used. Thus, there does not appear to be an obvious advantage to using averaging when modeling a BW slab since neither yields the correct behavior at the design frequency. (However, again note that we are not concerned with the specific location of the boundary, but rather just the behavior of the evanescent fields.)

In the PSTD scheme all field components are collocated and there is no transition layer at a material interface. Using the ADE method to implement the frequency dispersion of the BW material we observe that the nonreflecting wave occurs at the design frequency, as shown in Fig. 7. Because the wave vector magnitudes inside and outside the material are nearly equal (with slight discrepancy due to loss and a nonzero resonance frequency), the amplification of the evanescent wave inside the slab compensates for its free-space decay. The field amplitudes 


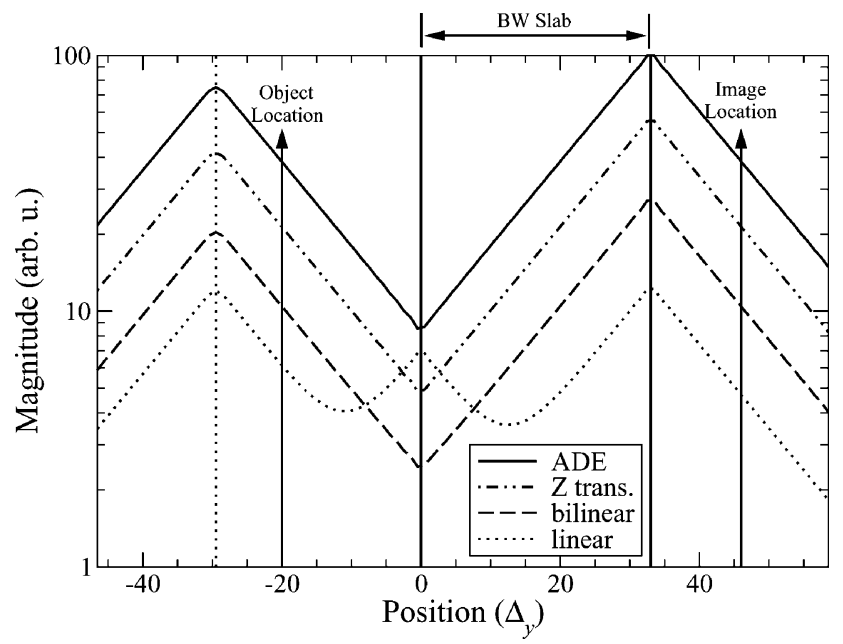

Fig. 8. Magnitude of the Fourier transform component at the design frequency using the PSTD scheme. The field is calculated using the ADE, Z-transform, and the bilinear and the linear frequency approximation methods. A reflected wave is clearly visible with the linear frequency approximation. The system parameters are given in the text. The lines for the different fields have been offset to allow qualitative comparison.

at the object location and its image location are within $0.5 \%$ of each other.

The examples in Figs. 3-6 are at a discretization level of $\lambda_{f} / 100$. Arguably, for either the averaged or abrupt boundary, this provides a transition layer thickness of only $\lambda_{f} / 200$ in the Yee scheme and yet it still produces a significant enough frequency shift for the nonreflecting wave that realization of a perfect planar lens is essentially impossible. The analysis in [13] shows that in the limit of vanishing layer thickness the frequency of the nonreflecting wave goes to the design frequency in the continuous case. Nevertheless, for all practical discretization levels the influence of the transition layer remains significant. There have been reports of the Yee scheme being used to model evanescent fields in BW materials [33], [34], but the level of discretization that had to be used was on the order of 566 cells per propagating wavelength [35] which may be prohibitive for most applications.

In general all finite-difference schemes suffer numerical dispersion. The PSTD scheme is more computationally expensive than the Yee algorithm for the same level of discretization but suffers less numerical dispersion. The dispersive properties of the PSTD scheme have been discussed in the literature [18] (although the dispersion relation given in the literature only pertains to the case of an infinite grid and thus only holds approximately for finite grids). The dispersive properties for evanescent fields in FDTD simulations have also been discussed in the literature [36]. The fields being considered here are discretized such that numeric dispersion is not a significant concern. Thus the major difference between the schemes considered here is the treatment of the interface and one cannot attribute the superior results of the PSTD scheme to its superior dispersion.

\section{B. Comparison of Dispersion Implementations}

In Section III-C we discussed four different implementations of the frequency dependence of the permeability and permit- tivity. To compare their performance, we use each one in a simulation using the PSTD scheme at the design frequency, with the parameters given at the beginning of Section IV. In this configuration one expects the reflected wave at the first interface $(y=0)$ to vanish, as shown in Fig. 7. In Fig. 8 we show the electric field in these simulations. It is apparent that the ADE, the Z-transform, and the bilinear frequency approximation all give good results. No reflected wave is visible and upon inspection one finds that the magnitudes at the object and image location are essentially equal. When plotted without offset the three curves fall on top of one another. The linear frequency approximation on the other hand does show a significant amplitude for the reflected wave. The field at the image location is also much weaker than at the object location. This effect is probably due to the numerical phase error inherent in this method [32]. This shows that the use of the linear frequency approximation is problematic in simulations involving BW materials, while many of the other common dispersive material implementations work well.

\section{CONCLUSION}

We have studied the FDTD and the PSTD methods in the context of evanescent waves in the presence of a BW material. Our simulations verify that the predicted growth of the evanescent field inside the BW material, which is vital for imaging beyond the diffraction limit, does occur. We found that the Yee FDTD method suffers from a transition-layer effect due to the inherent staggered grid, even when using an averaging technique at the material interface, while the collocated grid used in the PSTD method avoids this numerical artifact. The choice of numerical technique to implement the frequency dependence of the permittivity and the permeability is also important for the correct modeling of the BW material.

Thus, we have shown that the PSTD method in concert with the ADE technique, the Z-transform technique, or the bilinear frequency approximation, allows one to accurately model the interaction of a BW slab with an evanescent wave.

\section{ACKNOWLEDGMENT}

The authors would like to thank C. L. Wagner of Washington State University for useful discussion.

\section{REFERENCES}

[1] V. G. Veselago, "The electrodynamics of substances with simultaneously negative values of $\varepsilon$ and $\mu$," Sov. Phys. Usp., vol. 10, no. 4, pp. 509-514, Jan.-Feb. 1968.

[2] R. A. Shelby, D. R. Smith, and S. Schultz, "Experimental verification of a negative index of refraction,' Science, vol. 292, no. 1, pp. 77-79, Apr. 2001.

[3] D. R. Smith, W. J. Padilla, D. C. Vier, S. C. Nemat-Nasser, and S. Schultz, "Composite medium with simultaneously negative permeability and permittivity," Phys. Rev. Lett., vol. 84, no. 18, pp. 4184-4187, May 2000.

[4] J. B. Pendry, "Negative refraction makes a perfect lens," Phys. Rev. Lett., vol. 85 , no. 18 , pp. 3966-3969, Oct. 2000.

[5] G. W. 't Hooft, "Comment on "Negative refraction makes a perfect lens"," Phys. Rev. Lett., vol. 87, no. 24, article 249701, Dec. 2001.

[6] J. B. Pendry, "Comment on "Negative refraction makes a perfect lens" - Reply," Phys. Rev. Lett., vol. 87, no. 24, article 249702, Dec. 2001.

[7] N. Garcia and M. Nieto-Vesperinas, "Left-handed materials do not make a perfect lens," Phys. Rev. Lett., vol. 88, no. 20, article 207403, May 2002. 
[8] G. Gómez-Santos, "Universal features of the time evolution of evanescent modes in a left-handed perfect lens," Phys. Rev. Lett., vol. 90, no. 7, article 077401, Feb. 2003.

[9] P. M. Valanju, R. M. Walser, and A. P. Valanju, "Wave refraction negative-index media: Always positive and very inhomogeneous," Phys. Rev. Lett., vol. 88, no. 18, article 187401, May 2002.

[10] J. B. Pendry and D. R. Smith, "Comment on "Wave refraction in negative-index media: Always positive and very inhomogeneous"," Phys. Rev. Lett., vol. 90, no. 2, article 029703, Jan. 2003.

[11] P. M. Valanju, R. M. Walser, and A. P. Valanju, "Comment on "Wave refraction in negative-index media: Always positive and very inhomogeneous" - Reply," Phys. Rev. Lett., vol. 90, no. 2, article 029704, Jan. 2003.

[12] A. Grbic and G. V. Eleftheriades, "Growing evanescent waves in negative-refractive-index transmission-line media," Appl. Phys. Lett., vol. 82, no. 12, pp. 1815-1817, Mar. 2003.

[13] M. W. Feise, P. J. Bevelacqua, and J. B. Schneider, "Effects of surface waves on the behavior of perfect lenses," Phys. Rev. B, vol. 66, no. 3, article 035113, July 2002.

[14] R. d. L. Kronig, "On the theory of dispersion of X-rays," J. Opt. Soc. Amer., vol. 12, no. 6, pp. 547-557, June 1926.

[15] H. A. Kramers, Estratto Dagli Atti del Congresso Internazionale de Fisici, vol. 2, Como, Italy, 1927, p. 545.

[16] K. S. Yee, "Numerical solution of initial boundary value problems involving Maxwell's equations in isotropic media," IEEE Trans. Antennas Propagat., vol. AP-14, pp. 302-307, May 1966.

[17] A. Taflove, Computational Electrodynamics: The Finite-Difference Time-Domain Method, 1st ed. Boston, MA: Artech House, 1995.

[18] Q. H. Liu, "The PSTD algorithm: A time-domain method requiring only two cells per wavelength," Microwave Opt. Technol. Lett., vol. 15, no. 3, pp. 158-165, June 1997.

[19] J.-P. Berenger, "A perfectly matched layer for the absorption of electromagnetic waves," J. Comput. Phys., vol. 114, pp. 185-200, 1994.

[20] Z. S. Sacks, D. M. Kingsland, R. Lee, and J.-F. Lee, "A perfectly matched anisotropic absorber for use as an absorbing boundary condition," IEEE Trans. Antennas Propagat., vol. 43, pp. 1460-1463, Dec. 1995.

[21] M. Kuzuoglu and R. Mittra, "Frequency dependence of the constitutive parameters of causal perfectly matched anisotropic absorbers," IEEE Microwave Guided Wave Lett., vol. 6, pp. 447-449, Dec. 1996.

[22] M. Celuch-Marcysiak and W. K. Gwarek, "Higher-order modeling of media interfaces for enhanced FDTD analysis of microwave circuits," in Proc. 24th Eur. Microwave Conf., Cannes, France, Sept. 1994, pp. $1530-1535$.

[23] K.-P. Hwang and A. C. Cangellaris, "Effective permittivities for secondorder accurate FDTD equations at dielectric interfaces," IEEE Microw. Wireless Components Lett., vol. 11, pp. 158-160, Apr. 2001.

[24] J. B. Schneider and R. J. Kruhlak, "Plane waves and planar boundaries in FDTD simulations," presented at the Proc. IEEE Antennas and Propagat. Soc. Int. Symp. and URSI Radio Sci. Meeting, Salt Lake City, UT, July 2000 .

[25] R. W. Ziolkowski and E. Heyman, "Wave propagation in media having negative permittivity and permeability," Phys. Rev. E, vol. 64, no. 5, article 056625, Oct. 2001.

[26] R. M. Joseph, S. C. Hagness, and A. Taflove, "Direct time integration of Maxwell's equations in linear dispersive media with absorption for scattering and propagation of femtosecond electromagnetic pulses," Opt. Lett., vol. 16, no. 18, pp. 1412-1414, Sept. 1991.

[27] R. J. Luebbers, F. Hunsberger, and K. S. Kunz, "A frequency-dependent finite-difference time-domain formulation for transient propagation in plasma," IEEE Trans. Antennas Propagat., vol. 39, pp. 29-34, Jan. 1991.
[28] D. F. Kelley and R. J. Luebbers, "Piecewise linear recursive convolution for dispersive media using FDTD," IEEE Trans. Antennas Propagat., vol. 44, pp. 792-797, June 1996.

[29] D. M. Sullivan, "Frequency-dependent FDTD methods using Z transforms," IEEE Trans. Antennas Propagat., vol. 40, pp. 1223-1230, Oct. 1992.

[30] — "Z-transform theory and the FDTD method," IEEE Trans. Antennas Propagat., vol. 44, pp. 28-34, Jan. 1996.

[31] — Electromagnetic Simulation using the FDTD Method. New York: IEEE Press, 2000, IEEE Press series on RF and Microwave Technology.

[32] C. Hulse and A. Knoesen, "Dispersive models for the finite-difference time-domain method: Design, analysis, and implementation," J. Opt. Soc. Amer. A, vol. 11, no. 6, pp. 1802-1811, June 1994.

[33] X. S. Rao and C. K. Ong, "Amplification of evanescent waves in a lossy left-handed material slab," Phys. Rev. B, vol. 68, no. 11, article 113103, Sept. 2003.

[34] - "Subwavelength imaging by a left-handed material superlens," Phys. Rev. E, vol. 68, no. 6, article 067601, Dec. 2003.

[35] X. S. Rao, Personal Communication, Oct. 2003.

[36] J. B. Schneider and R. J. Kruhlak, "Dispersion of homogeneous and inhomogeneous waves in the Yee finite-difference time-domain grid," IEEE Trans. Microwave Theory Tech., vol. 49, pp. 280-287, Feb. 2001.

Michael W. Feise received the M.S. and Ph.D. degree in physics from Washington State University, Pullman, in 1999 and 2001, respectively.

From 2001 to 2003, he was a Research Associate with the School of Electrical Engineering and Computer Science, Washington State University. In 2003, he became a Research Fellow in the Nonlinear Physics Centre, Research School of Physical Sciences and Engineering, Australian National University. His research interests include electromagnetic and acoustic wave propagation and their linear and nonlinear interaction with matter, as well as $\mathrm{THz}$ radiation, and low-dimensional semiconductor devices.

John B. Schneider (M'00) received the B.S. degree in electrical engineering from Tulane University, New Orleans, LA, and the M.S. and Ph.D. degrees in electrical engineering from the University of Washington, Seattle.

$\mathrm{He}$ is presently an Associate Professor in the School of Electrical Engineering and Computer Science, Washington State University, Pullman. His research interests include the use of computational methods to analyze acoustic, elastic, and electromagnetic wave propagation.

Peter J. Bevelacqua received the B.S. degree in electrical engineering (summa cum laude) from Washington State University, Pullman, in 2002. He is currently working toward the Ph.D. degree at Stanford University, Stanford, CA.

In 2002, he worked as an Intern at Sandia National Labs, Albuquerque, New Mexico.

Mr. Bevelacqua was awarded a National Science Foundation Fellowship in 2003. 